\title{
Surmounting fluctuating barriers
}

\author{
The idea that particles can surmount the walls of potential wells to escape from them is so familiar that the \\ complexity of attempts to broaden the problem is a surprise.
}

Even those whose belief in quantum mechanics is of the formal kind, like that of those who give Einstein full marks for precocity, but who have never sensed that curvature can stand proxy for gravitational force, are often persuaded by one revelation of the elementary textbooks: particles trapped (in the classical sense) in a potential well can, in reality, leak out. The emission of $\alpha$-particles from neutron-rich radioactive nuclei is a practical demonstration of the phenomenon; classically, centripetal nuclear forces would prevent the $\alpha$-particles from escaping, but in quantum mechanics, they can tunnel through the potential energy barrier.

An even more compelling proof that quantum mechanics describes the real world (if only because the constituency of chemists is probably larger than any other) is the demonstration (rigorously by Kramers, only in 1940) that the rate at which particles escape a potential barrier is exponentially related to its height, say $E$, and the temperature at which the escaping particles are maintained, say $T$, by a factor in which the exponent is $-(E / k T)$, where $k$ is Boltzmann's constant.

Chemists are encouraged because this is precisely the dependence (in matters such as reaction rates) discovered empirically by Arrhenius in the nineteenth century. One minor difficulty is that classical kinetic theory predicts the same kind of dependence; the essence of quantum tunnelling is that even particles with insufficient energy can tunnel through barriers that would classically constrain them.

Nevertheless the idea that reaction rates are regulated by the ease with which quantum systems can surmount potential barriers has become generally acceptable. The standard textbook picture is that of two regions of space separated by a fixed potential barrier. The problem is to tell the probability that particles on one side of the barrier, whatever their energy (which may vary), will escape to the other.

So far, so good. But what will happen if the potential barrier is not fixed for all time, but rather is itself variable? Only a little imagination is required to suggest a host of circumstances in which these circumstances might apply. The potential barrier to the disassociation of a molecule, for example, may depend on the geometrical positions of atoms not directly involved in what may be simply the stretching of a chemical bond to destruction. Or, as much to the point, an insulating layer separating two semiconducting layers may be subject to a bias that affects the ease with which electrons can tunnel through it. The fluctuating barrier is a concept whose time appears to have come.

Charles R. Doering and Jonathan C. Gadoua from Clarkson University at Potsdam, New York, have arranged just that (Phys. Rev. Lett. 69, 2,318; 19 October 1992). Their calculation is strictly classical, with no quantum tunnelling, and might apply to the particles of a perfect gas enclosed in a one-dimensional box that itself encloses the potential barrier. To make the problem tractable (and there is a long way to go), the authors consider the most stylized of all possible potential barriers - a simple symmetrical triangular profile that puts a maximum of the potential energy at the centre of the one-dimensional box.

The problem is really a problem in diffusion. If the box contains an ensemble of freely moving particles which is nevertheless sufficiently dense to ensure that the movement of each is impeded by its neighbours, its momentum will on the average be proportional to the slope of the potential barrier (the driving force), but will be augmented by the impulsive forces from collisions with other particles. Although these are assumed to be entirely random in magnitude and direction, subject to constituting white noise, they may well be sufficient to carry a particular particle over the peak of the triangular barrier.

That is the classical problem-setting for an ensemble of particles in a box with a fixed potential barrier. How to make the barrier fluctuate? Doering and Gadoua use the simplest possible recipe. They allow for two alternative triangular potential barriers in their one-dimensional box, but let its height switch from one value to another at times that are inherently unpredictable, but which are determined by a single time-constant which is numerically defined.

Where is this leading them (or us, for that matter)? To a counter-intuitive conclusion, that is where. What would one expect? Suppose the parameter that matters is the average time it takes a particle to cross the barrier (or to reach its highest point, which is the same thing).

If the fluctuations between one form of the barrier and the other are relatively slow (compared with the characteristic timescale of the particles), the average crossing time would be the mean of the average crossing times over the two barriers separately; on the average, each should be in place for the same length of time, while the edge effects in which the dynamics appropriate to one condition gave particles extra freedom in the other would shrink to zero. But if, conversely, the barrier fluctuations are relatively fast, the edge effects are everything and the particles behave as if they are exposed to an average of the two extreme potentials. Because the crossing time is related to an exponential function of the energy, the two averages are not the same at all.

In many ways, this is the simplest version of the fluctuating barrier problem there could be. (The notion that the intervals between switches from one barrier to the other should be random is not a complication, but the opposite, allowing the parametrization, as the saying goes, of the state of the system by the ratio of two time constants.) The authors have carried out Monte Carlo simulations of what happens and have reported analytical results where they are attainable (by the aid of a what is no doubt a formidable amount of unrecorded algebra).

The upshot is that naive expectations are confirmed when the switching time between one barrier and the other is either very long or very short. But, in between, particles almost always more easily surmount the fluctuating barrier than simple averaging would suggest. Moreover, there seems to be a resonance phenomenon. Given some match between the switching time and that taken by a particle to surmount the barrier, the ease of transition is even greater than expected. Anthropomorphically speaking, this may mean no more than that the particles that more easily get through are those that are clever enough to wait until the barrier is lowered.

The importance of these calculation probably lies in the analysis of the stability of truly complex systems, but those with other interests will remark on two features of this calculations. First, it is remarkable, in this day and age, how simple are the systems that form the substrate of people's elaborate calculations. That is one measure of how far there is to go to the treatment of the real world. And then there is the question of when it will be possible to treat real quantum systems. On that score too, there is a rough road ahead.

John Maddox 